\title{
Uppsala NLP at SemEval-2021 Task 2: Multilingual Language Models for Fine-tuning and Feature Extraction in Word-in-Context Disambiguation
}

\author{
Huiling You, Xingran Zhu, and Sara Stymne \\ Uppsala University \\ Uppsala, Sweden \\ \{Huiling.You.7480,Xingran.Zhu.2781\}estudent.uu.se \\ sara.stymnedlingfil.uu.se
}

\begin{abstract}
We describe the Uppsala NLP submission to SemEval-2021 Task 2 on multilingual and cross-lingual word-in-context disambiguation. We explore the usefulness of three pre-trained multilingual language models, XLM-RoBERTa (XLMR), Multilingual BERT (mBERT) and multilingual distilled BERT (mDistilBERT). We compare these three models in two setups, fine-tuning and as feature extractors. In the second case we also experiment with using dependency-based information. We find that fine-tuning is better than feature extraction. XLMR performs better than mBERT in the cross-lingual setting both with fine-tuning and feature extraction, whereas these two models give a similar performance in the multilingual setting. mDistilBERT performs poorly with fine-tuning but gives similar results to the other models when used as a feature extractor. We submitted our two best systems, fine-tuned with XLMR and mBERT.
\end{abstract}

\section{Introduction}

SemEval-2021 Task 2: Multilingual and Crosslingual Word-in-Context Disambiguation (MCLWiC) (Martelli et al., 2021) is an extension from WiC (Pilehvar and Camacho-Collados, 2019), a shared task at the IJCAI-19 SemDeep workshop (SemDeep-5). WiC was proposed as a benchmark to evaluate context-sensitive word representations. The WiC dataset ${ }^{1}$ consists of a list of English sentence-pairs. Each sentence-pair has a target word, and the task is to determine whether the target word is used in the same meaning or different meanings in the two sentences, thus as a binary classification task. MCL-WiC extends WiC to multilingual and cross-lingual datasets, ${ }^{2}$ and covers 5

\footnotetext{
${ }^{1}$ https://pilehvar.github.io/wic/.

${ }^{2}$ https://github.com/SapienzaNLP/ mcl-wic
}

\begin{tabular}{|l|c|}
\hline Example & Label \\
\hline $\begin{array}{l}\text { The cat chases after the mouse. } \\
\text { Click the right mouse button. }\end{array}$ & $\mathrm{F}$ \\
\hline $\begin{array}{l}\text { The cat chases after the mouse. } \\
\text { La souris mange le fromage. } \\
\text { ('The mouse eats the cheese') }\end{array}$ & $\mathrm{T}$ \\
\hline
\end{tabular}

Table 1: Examples for multilingual (top) and crosslingual (bottom) word-in-context disambiguation.

languages: Arabic, Chinese, English, French, and Russian. The MCL-WiC task is also framed as a binary classification task: given a sentence-pair with a target word, either in the same language or in different languages, the goal is to determine whether the target word is used in the same meaning or in different meanings. Table 1 shows two example sentence pairs where the target word (mouse) has either an 'animal' or a 'computer' sense. In the multilingual setting, the two sentences are from the same language. In the cross-lingual setting, the two sentences are from different languages, English and one of the other four languages. Training data is only available for English-English, effectively leading to a zero-shot setting for the other languages.

Our main interest is to investigate the usefulness of pre-trained multilingual language models (LMs) in this MCL-WiC task, without resorting to sense inventories, dictionaries, or other resources. As our main method, we fine-tune the language models with a span classification head. We also experiment with using the multilingual language models as feature extractors, extracting contextual embeddings for the target word. In this setting, we also add information about syntactical dependency (i.e. head words and dependent words), with the intuition that it can contain relevant contextual information for disambiguation, as in Figure 1, 
where the head words chases and button could help in disambiguating mouse. We compare three different LMs: XLM-RoBERTa (XLMR), multilingual BERT (mBERT) and multilingual distilled BERT (mDistilBERT).

We show that the fine-tuned models are stronger than any of the models based on feature extraction, by a large margin. XLMR is stronger than mBERT in the cross-lingual setting both with finetuning and feature extraction. mDistilBERT gives poor results with fine-tuning, but is competitive to the other LMs when used for feature extraction. Adding dependency syntax to our feature extraction method led to mixed results. We submitted our two strongest systems to the shared task, those fine-tuned with XLMR and mBERT.

\section{Related Work}

In $\mathrm{WiC}$ at SemDeep-5, many participating systems capitalized on contextualized word representations. The LMMS (Language Modelling Makes Sense) system by Loureiro and Jorge (2019) used word embeddings from BERT, together with sense embeddings from WordNet 3.0 (Marciniak, 2020). Ansell et al. (2019) used the contextualized representations from ELMo (Peters et al., 2018) and trained a separate classification model. Soler et al. (2019) experimented with several contextualized representations and used cosine similarity to measure word similarities. Wang et al. (2019) included $\mathrm{WiC}$ as one of the tasks in the proposed SuperGLUE benchmark, with the approach of fine-tuning BERT. At the end of the $\mathrm{WiC}$ evaluation period, the best result was achieved by Wang et al. (2019) with an accuracy of $68.36 \%$, while human-level performance is $80 \%$, as provided by the dataset curators.

Scarlini et al. (2020) recently proposed SensEm$\mathrm{BERT}^{3}$, a knowledge-based approach to sense embeddings for multiple languages. An important source for building SenseEmBERT is the contextualized representations from a pretrained language model. They experimented with SensEmBERT on both English and multilingual word sense disambiguation (WSD) tasks, and showed that SensEmBERT is able to achieve state-of-the-art result on both English and multilingual WSD datasets.

\footnotetext{
${ }^{3}$ http: / / sensembert.org/
}

\section{Multilingual Language Models}

\subsection{XLMR}

XLMR (XLM-RoBERTa) is a scaled cross-lingual sentence encoder (Conneau et al., 2020), which is trained on 2.5T of data obtained from Common Crawl that covers more than 100 languages. XLMR has achieved state-of-the-art results on various cross-lingual NLP tasks.

\subsection{MBERT}

mBERT (multilingual BERT) is pre-trained on the largest Wikipedias (Libovický et al., 2019). It is a multilingual extension of BERT (Devlin et al., 2019) that provides word and sentence representations for 104 languages, which has been shown to be capable of clustering polysemic words into distinct sense regions in the embedding space (Wiedemann et al., 2019).

\section{3 mDistilBERT}

mDistilBERT (multilingual distilled BERT) is a light Transformer trained by distilling mBERT (Sanh et al., 2019), which reduces the number of parameters in mBERT by $40 \%$, increases the speed by $60 \%$, and retains over $97 \%$ of mBERT's performance.

\subsection{Sub-word models}

XLMR, mBERT, and mDistilBERT all use subword models (Wu et al., 2016; Kudo and Richardson, 2018), so the target word is usually represented by several sub-tokens. For example, given "qualify" as target word, it will be represented by "quali" and "fy" in XLMR. mBERT and mDistilBERT use a WordPiece model with a vocabulary size of 119,447 and XMLR use a SentencePiece model with a vocabulary size of 250,002 . In our work, when the target word is represented by multiple sub-words, we use the averaged embedding as feature vector for the target word. ${ }^{4}$

\section{System Description}

We use the pre-trained language models in two different ways: for fine-tuning (Section 4.1) and as feature extractors (Section 4.2 - 4.3). Depending on whether feature transformation is involved, the features extracted can be further categorized into target

\footnotetext{
${ }^{4}$ We also explored summing sub-words, which gave similar results to averaging.
} 


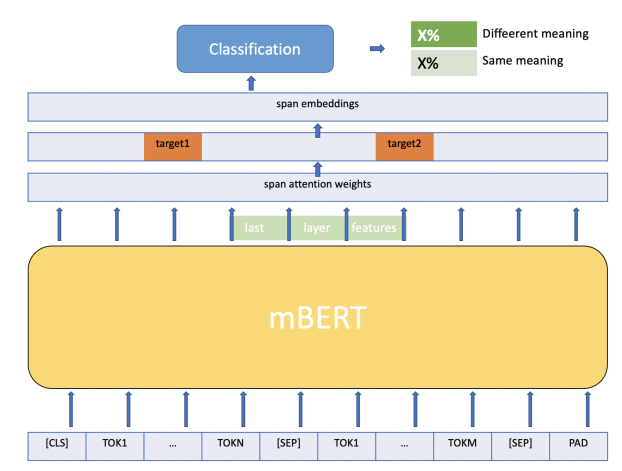

Figure 1: Model Structure of Fine-tuning mBERT

word embeddings (Section 4.2) and dependencybased syntax-incorporated word embeddings (Section 4.3). In the following sub-sections, we describe the three systems respectively. Due to time constraints we did not use XLMR in the systems with feature extraction.

\subsection{Fine-Tuning}

The fine-tuning setup follows the architecture designed by Wang et al. (2019), ${ }^{5}$ but extends to datasets in multiple languages. A span classification head is stacked on top of pre-trained language models, and attends only to the target words. The span classification head consists of a span attention extractor and a classifier. The span attention extractor is responsible for extracting the span embeddings, namely the target words embeddings. First, the unnormalized attention score of each token of the input document is computed. Span attention scores are the normalized scores of all tokens inside the span. Given the attention distributions over spans, each span gets a weighted representation of the last-layer hidden states of either mBERT, mDistilBERT or XLMR.

In this task, only the two target word spans will be returned, by masking out the rest of input. The attended span embeddings are then passed to the classifier, a linear transformation layer, to produce the output logits, which have a dimension of two, since there are only two labels (True or False). Figure 1 exemplifies the model structure when finetuning mBERT. The same structure also applies to XLMR and mDistilBERT.

\footnotetext{
${ }^{5}$ The package for SuperGLUE tasks is available at https : //github.com/nyu-mll/jiant
}

\subsection{Target Words Embeddings}

In this setup, the multilingual language models serve as pure feature extractors, to get target word embeddings from last-layer hidden states. The input sample of a sentence-pair will then be the concatenation of the pair of target word embeddings.

We feed the two sentences separately to the models, and concatenate the embeddings for the two target words. ${ }^{6}$ The extracted feature vectors are then fed to a classifier to perform the binary classification task. We experimented with two classifiers, logistic regression (LR) and a multi-layer perceptron (MLP).

\subsection{Dependency-based Syntax-Incorporated Embeddings}

In this setup we ran a limited number of experiments. Only four languages (English, French, Chinese, and Russian) ${ }^{7}$ and two pre-trained language models (mBERT and mDistilBERT) are explored.

The reasoning behind using syntax information to improve WiC classification results is as following. Given a pair of sentences, where the first sentence is "The cat chases after the mouse", and the second one is "Click the right mouse button", the target word mouse has different head words: in the first sentence, the singular verb chases is the head word, whereas in the second sentence, the noun button is the head word. Since it is more natural for a real mouse (as a small rodent) to be chased by its predators than to be related to a button, while in contrast, it is more common for a computer mouse (as a hand-held pointing device) to have a button than to be chased, the head words therefore reveal information on different contexts of the target word. The same reasoning applies to dependent words as well.

First, each sentence is parsed using the spaCy dependency parser, ${ }^{8}$ from which we extract the target word, its head word, and its dependent word(s). Next, the sentence is passed to mBERT or mDistilBERT, and the corresponding target word embedding, head word embedding, and dependent

\footnotetext{
${ }^{6} \mathrm{We}$ also experimented with concatenating the two sentences before feeding it to the LM, which gave slightly better results in some experiments. For consistency among all experiments we do not report these results.

${ }^{7}$ The latest version of spaCy (3.0.0), which is the dependency parsing library used in this work, does not support dependency parsing for Arabic, thus we do not run experiments on Arabic in this setup.

${ }^{8}$ https: / / spacy.io/usage/ linguistic-features\#dependency-parse
} 


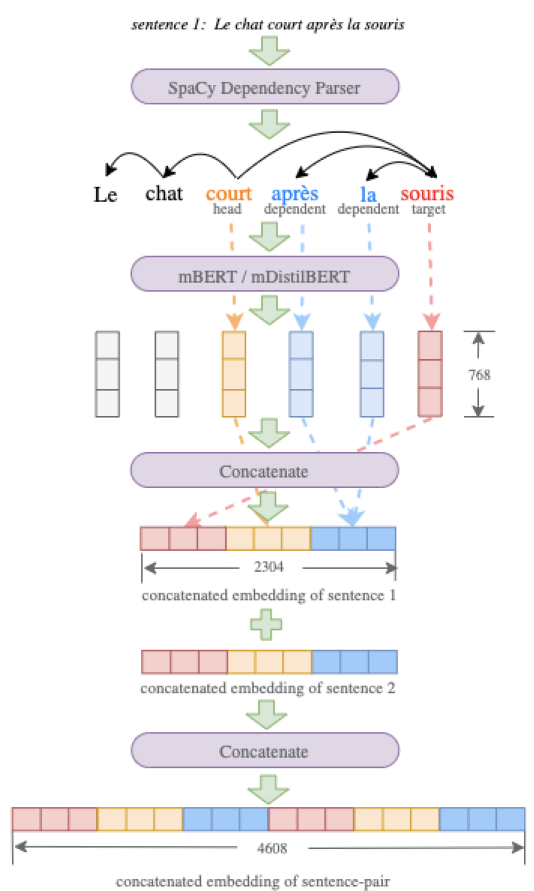

Figure 2: Construct a dependency-based syntaxincorporated embedding for a sentence-pair

word embedding(s) are retrieved, and concatenated. Note that if the target word has no head or dependent word, the null token embedding ${ }^{9}$ is used instead; if the target word has more than one dependent word, all dependent word embeddings are summed element-wise. ${ }^{10}$ Finally, the concatenated embeddings of two constituent sentences are further concatenated to form the sample feature vector of the sentence-pair, which is then fed to an MLP.

Figure 2 illustrates the process of constructing one such dependency-based syntax-incorporated embedding for a sentence-pair, of which the first sentence is Le chat court après la souris. The default embedding size of $\mathrm{mBERT} / \mathrm{mDistilBERT}$ is 768. The sizes of different concatenated embeddings are shown in Figure 2. Again, we experimented with two classifiers, logistic regression and a multi-layer perceptron.

\section{Experimental Setup}

Dataset Only the datasets provided by SemEval2021 Task 2 are used, see Table 2. All systems are trained on the English set, the multilingual development sets are used during development, and the

\footnotetext{
${ }^{9}$ That is, simply feeding the word null into $\mathrm{mBERT} / \mathrm{mDistilBERT}$ and using the generated embedding directly.

${ }^{10} \mathrm{We}$ also explored averaging the dependent word embeddings, which gave equivalent results to summing.
}

\begin{tabular}{l|ccc}
\hline & Train & Dev & Test \\
\hline en-en & 8000 & 500 & 1000 \\
\hline ar-ar & - & 500 & 1000 \\
fr-fr & - & 500 & 1000 \\
ru-ru & - & 500 & 1000 \\
zh-zh & - & 500 & 1000 \\
\hline en-ar & - & - & 1000 \\
en-fr & - & - & 1000 \\
en-ru & - & - & 1000 \\
en-zh & - & - & 1000 \\
\hline
\end{tabular}

Table 2: SemEval-2021 Task 2 Datasets. At development time, we only use half of the provided size (1000) of each dev set.

systems are tested on the multilingual and crosslingual test sets.

Fine-tuning The three multilingual language models (mBERT, mDistilBERT, XLMR) are finetuned for three iterations, with batch size of 32 , learning rate of $1 \mathrm{e}-5$, and parameters optimized with AdamW (Loshchilov and Hutter, 2018), provided by Huggingface's Transformers library ${ }^{11}$.

Logistic Regression All logistic regression (referred to as "LR" in the following sections) models are trained for 150 iterations, with batch size of 32, learning rate of 0.0025 and parameters optimized with standard stochastic gradient descent (SGD).

MLP All MLP models are 2-layer and follow the architecture suggested by Du et al. (2019), outputing classification label based on the probability:

$$
\mathbf{p}=\operatorname{softmax}\left(L_{2}\left(\operatorname{ReLU}\left(L_{1}(\mathbf{e})\right)\right)\right)
$$

where $\mathbf{e}$ is in the input embedding, $L_{i}(\mathbf{x})=\mathbf{W}_{i} \mathbf{x}+$ $\mathbf{b}_{i}$ are fully-connected layers, $\mathbf{W}_{1} \in \mathbb{R}^{H \times H}$ and $\mathbf{W}_{2} \in \mathbb{R}^{2 \times H}$ are layer parameter matrices, and $H$ is the input embedding size. All MLP models are trained for maximum 200 iterations, with learning rate of 0.001 and parameters optimized with Adam $\left(\beta_{1}=0.9, \beta_{2}=0.999\right)($ Kingma and $\mathrm{Ba}, 2015)$.

Language Model We use the base version of all multilingual language models, with 12 layers, 12 attention heads, and hidden dimension of 768. Due to time constraints we did not use XLMR in the systems with feature extraction and an MLP.

\footnotetext{
${ }^{11}$ https://huggingface.co/transformers/
} 


\begin{tabular}{|c|c|c|c|c|c|c|c|c|c|c|}
\hline & System & en-en & zh-zh & fr-fr & ru-ru & ar-ar & en-zh & en-fr & en-ru & en-ar \\
\hline \multirow{3}{*}{ Fine-tune } & XLMR & $84.5 \%$ & $78.3 \%$ & $76.7 \%$ & $73.1 \%$ & $75.1 \%$ & $66.3 \%$ & $70.9 \%$ & $73.6 \%$ & $65.2 \%$ \\
\hline & mBERT & $82.9 \%$ & $76.2 \%$ & $80.3 \%$ & $73.6 \%$ & $75.6 \%$ & $62.2 \%$ & $66.3 \%$ & $63.1 \%$ & $59.4 \%$ \\
\hline & mDistilBERT & $75.5 \%$ & $68.0 \%$ & $66.8 \%$ & $64.8 \%$ & $68.9 \%$ & $51.8 \%$ & $53.4 \%$ & $51.9 \%$ & $50.9 \%$ \\
\hline \multirow{7}{*}{$\begin{array}{l}\text { Feature } \\
\text { Extractor }\end{array}$} & XLMR + LR & $53.9 \%$ & $55.4 \%$ & $54.8 \%$ & $57.2 \%$ & $53.0 \%$ & $58.2 \%$ & $55.8 \%$ & $55.4 \%$ & $54.7 \%$ \\
\hline & mBERT + LR & $53.4 \%$ & $53.5 \%$ & $49.7 \%$ & $51.7 \%$ & $53.1 \%$ & $52.0 \%$ & $52.8 \%$ & $52.8 \%$ & $51.1 \%$ \\
\hline & mDistilBERT + LR & $55.7 \%$ & $50.5 \%$ & $52.6 \%$ & $52.5 \%$ & $51.9 \%$ & $54.0 \%$ & $52.5 \%$ & $52.0 \%$ & $51.6 \%$ \\
\hline & mBERT + MLP & $67.7 \%$ & $51.4 \%$ & $57.6 \%$ & $54.2 \%$ & $54.0 \%$ & $47.4 \%$ & $62.6 \%$ & $55.6 \%$ & $53.2 \%$ \\
\hline & mDistilBERT + MLP & $66.6 \%$ & $59.1 \%$ & $59.8 \%$ & $61.8 \%$ & $56.0 \%$ & $48.2 \%$ & $63.2 \%$ & $57.4 \%$ & $52.3 \%$ \\
\hline & mBERT + Syntax + MLP & $61.4 \%$ & $52.7 \%$ & $57.6 \%$ & $57.0 \%$ & - & $53.4 \%$ & $57.8 \%$ & $55.6 \%$ & - \\
\hline & mDistilBERT + Syntax + MLP & $67.0 \%$ & $56.6 \%$ & $58.2 \%$ & $57.6 \%$ & - & $54.0 \%$ & $57.2 \%$ & $56.2 \%$ & - \\
\hline
\end{tabular}

Table 3: System results on test sets. At task evaluation time, two fine-tuned systems were submitted, mBERT and XLMR; other systems were tested at post-evaluation time.

\section{Results and Analysis}

The evaluation results on the test sets are shown in Table 3. We can see that the fine-tuning approach is preferable to the feature extraction approach. All feature extraction variants fall behind the fine-tuned systems by a large margin. In many cases the systems based on feature extraction is just over chance performance $(50 \%)$, and in a few cases it is even below it.

Among the fine-tuned systems, XLMR and mBERT give the best results, whereas mDistilBERT falls behind by quite a large margin in most cases, in several cases by more than 10 percentage points. The performance of mDistilBERT is especially weak in the cross-lingual setting. XLMR gives the best results for all cross-lingual language pairs, with an improvement over mBERT of 4.110.5 percentage points. The improvement is largest for English-Russian. For the multilingual setting, the difference between mBERT and XLMR is smaller with at most 3.6 percentage points. XLMR gives the best score in two cases and mBERT in three cases.

Among the systems with feature extraction, the relative performance of the three sets of contextual embeddings differ from the fine-tuning. Here, mDistilBERT are competitive to the other two embeddings. We only use XLMR with LR, and again, we see that it gives the best performance in the cross-lingual setting among all systems with LR, just as with fine-tuning. In the multilingual setting, XLMR is also strong, having the best result for three out of five languages. Compared to finetuning, mDistilBERT performs surprisingly well here. It is on par or better than mBERT in most cases across all settings.

Comparing the different architectures used with the feature extraction strategy, we see that using an MLP is preferable to LR, leading to large improvements in most cases. An exception is
English-Chinese, where the MLP without syntax performs worse than LR. For English-French on the other hand, the MLP outperforms LR by around 10 percentage points, whereas we see small improvements for English-Russian. Finally, the addition of syntax leads to mixed results. For the English-Chinese system, we see large improvements, whereas we see the opposite for EnglishFrench. For English-Russian as well as for all multilingual systems, the differences are overall smaller.

We also note that the performance is stronger for English-English than for the other languages in most settings. This is expected, since we only have English-English training data. A notable exception is for LR, where English-English performs considerably worse than in all other settings and is on par with the other languages in the same setting. With fine-tuning we overall see stronger results in the multilingual setting, than in the cross-lingual setting, where we mix language pairs. We do not see this difference for our feature extraction systems, however.

\section{Conclusion and Future Work}

We have investigated the use of three large language models for multilingual and cross-lingual word-in-context disambiguation. We found that fine-tuning the language models is preferable to using them as feature extractors either for an MLP or for logistic regression. Trying to add dependencybased syntax information in the MLP gave mixed results. We also found that XLMR performed better than mBERT in the cross-lingual setting, both with fine-tuning and feature extraction, whereas the two models had a more similar performance in the multilingual setting. mDistilBERT did not perform well with fine-tuning, but was competitive to the other models in the feature extraction setting. We submitted our two best systems, fine-tuning with 
XLMR and mBERT to the shared task.

The fact that XLMR performs better than mBERT in the cross-lingual setting seems to indicate that it has a better representation of words across languages than mBERT and mDistilBERT. We think it would be worth investigating this hypothesis in more detail. XLMR and mBERT also use different sub-word models and another research direction is to explore the impact of this difference. We would also like to investigate the effect of using representations from different layers of the pretrained multilingual language models.

\section{References}

Alan Ansell, Felipe Bravo-Marquez, and Bernhard Pfahringer. 2019. An ELMo-inspired approach to SemDeep-5's word-in-context task. In Proceedings of the 5th Workshop on Semantic Deep Learning (SemDeep-5), pages 21-25, Macau, China. Association for Computational Linguistics.

Alexis Conneau, Kartikay Khandelwal, Naman Goyal, Vishrav Chaudhary, Guillaume Wenzek, Francisco Guzmán, Edouard Grave, Myle Ott, Luke Zettlemoyer, and Veselin Stoyanov. 2020. Unsupervised cross-lingual representation learning at scale. In Proceedings of the 58th Annual Meeting of the Association for Computational Linguistics, pages 84408451, Online. Association for Computational Linguistics.

Jacob Devlin, Ming-Wei Chang, Kenton Lee, and Kristina Toutanova. 2019. BERT: Pre-training of deep bidirectional transformers for language understanding. In Proceedings of the 2019 Conference of the North American Chapter of the Association for Computational Linguistics: Human Language Technologies, Volume 1 (Long and Short Papers), pages 4171-4186, Minneapolis, Minnesota. Association for Computational Linguistics.

Jiaju Du, Fanchao Qi, and Maosong Sun. 2019. Using BERT for word sense disambiguation. arXiv:1909.08358 [cs.CL].

Diederik Kingma and Jimmy Ba. 2015. Adam: A method for stochastic optimization. In Proceedings of the 3rd International Conference on Learning Representations, ICLR, San Diego, CA, USA.

Taku Kudo and John Richardson. 2018. Sentencepiece: A simple and language independent subword tokenizer and detokenizer for neural text processing. In Proceedings of the 2018 Conference on Empirical Methods in Natural Language Processing: System Demonstrations, pages 66-71.

Jindřich Libovický, Rudolf Rosa, and Alexander Fraser. 2019. How language-neutral is multilingual bert? arXiv:1911.03310 [cs.CL].
Ilya Loshchilov and Frank Hutter. 2018. Decoupled weight decay regularization. In Proceedings of the International Conference on Learning Representations (ICLR), Vancouver, Canada.

Daniel Loureiro and Alípio Jorge. 2019. Language modelling makes sense: Propagating representations through WordNet for full-coverage word sense disambiguation. In Proceedings of the 57th Annual Meeting of the Association for Computational Linguistics, pages 5682-5691, Florence, Italy. Association for Computational Linguistics.

Jacek Marciniak. 2020. Wordnet as a backbone of domain and application conceptualizations in systems with multimodal data. In Proceedings of the LREC 2020 Workshop on Multimodal Wordnets (MMW2020), pages 25-32, Marseille, France. The European Language Resources Association (ELRA).

Federico Martelli, Najla Kalach, Gabriele Tola, and Roberto Navigli. 2021. SemEval-2021 Task 2: Multilingual and Cross-lingual Word-in-Context Disambiguation (MCL-WiC). In Proceedings of the Fifteenth Workshop on Semantic Evaluation (SemEval2021).

Matthew Peters, Mark Neumann, Mohit Iyyer, Matt Gardner, Christopher Clark, Kenton Lee, and Luke Zettlemoyer. 2018. Deep contextualized word representations. In Proceedings of the 2018 Conference of the North American Chapter of the Association for Computational Linguistics: Human Language Technologies, Volume 1 (Long Papers), pages 22272237.

Mohammad Taher Pilehvar and Jose CamachoCollados. 2019. WiC: the word-in-context dataset for evaluating context-sensitive meaning representations. In Proceedings of the 2019 Conference of the North American Chapter of the Association for Computational Linguistics: Human Language Technologies, Volume 1 (Long and Short Papers), pages 1267-1273, Minneapolis, Minnesota. Association for Computational Linguistics.

Victor Sanh, Lysandre Debut, Julien Chaumond, and Thomas Wolf. 2019. DistilBERT, a distilled version of BERT: smaller, faster, cheaper and lighter. In Proceedings of The 5th Workshop on Energy Efficient Machine Learning and Cognitive Computing, Vancouver, Canada.

Bianca Scarlini, Tommaso Pasini, and Roberto Navigli. 2020. Sensembert: Context-enhanced sense embeddings for multilingual word sense disambiguation. In $A A A I$, pages $8758-8765$.

Aina Garí Soler, Marianna Apidianaki, and Alexandre Allauzen. 2019. Limsi-multisem at the ijcai semdeep-5 wic challenge: Context representations for word usage similarity estimation. In Proceedings of the 5th Workshop on Semantic Deep Learning (SemDeep-5), pages 6-11. 
Alex Wang, Yada Pruksachatkun, Nikita Nangia, Amanpreet Singh, Julian Michael, Felix Hill, Omer Levy, and Samuel Bowman. 2019. Superglue: A stickier benchmark for general-purpose language understanding systems. In Advances in Neural Information Processing Systems, pages 3266-3280.

Gregor Wiedemann, Steffen Remus, Avi Chawla, and Chris Biemann. 2019. Does BERT make any sense? interpretable word sense disambiguation with contextualized embeddings. In Proceedings of the 15th Conference on Natural Language Processing (KONVENS 2019): Long Papers, pages 161-170, Erlangen, Germany.

Yonghui Wu, Mike Schuster, Zhifeng Chen, Quoc V Le, Mohammad Norouzi, Wolfgang Macherey, Maxim Krikun, Yuan Cao, Qin Gao, Klaus Macherey, et al. 2016. Google's neural machine translation system: Bridging the gap between human and machine translation. arXiv preprint arXiv:1609.08144. 